\title{
A comparison of dispersion and Markov constants
}

\author{
by
}

Amitabha Tripathi (Fairmont, W.Va.)

0. Introduction. Let $\left\{x_{n}\right\}$ be a sequence of numbers, $0 \leq x_{n} \leq 1$. In [3], H. Niederreiter introduced a measure of denseness of such a sequence as follows: For each $N \geq 1$, let

$$
d_{N}=\sup _{0 \leq x \leq 1} \min _{1 \leq n \leq N}\left|x-x_{n}\right|
$$

and define

$$
D\left(\left\{x_{n}\right\}\right)=\limsup _{N \rightarrow \infty} N d_{N} .
$$

In particular, for irrational $\alpha$, the dispersion constant $D(\alpha)$ is defined by $D(\{n \alpha \bmod 1\})$. It is well known that, for irrational $\alpha$, the Markov constant $M(\alpha)$ is defined by

$$
M(\alpha)^{-1}=\liminf _{n \rightarrow \infty} n\|n \alpha\|,
$$

where $\|x\|$ denotes the distance from $x$ to the nearest integer.

In [3], Niederreiter asks if $M(\alpha)<M(\beta)$ implies $D(\alpha)<D(\beta)$. V. Drobot [1] has shown this to be false by producing a counterexample of two quadratic irrationals, both with continued fraction expansion with period length nine. In this paper, we classify some infinite families of pairs $(\alpha, \beta)$ of irrational numbers that satisfy $M(\alpha)<M(\beta)$ and $D(\alpha)>D(\beta)$.

We first outline the method of V. Drobot [1] to compute $D(\alpha)$ for quadratic irrationals $\alpha$. If $\alpha$ is a real irrational number with continued fraction expansion $\alpha=\left[a_{0} ; a_{1}, a_{2}, \ldots\right]$, let

$$
\lambda_{i}=\left[0 ; a_{i}, a_{i-1}, \ldots, a_{1}\right], \quad \Lambda_{i}=\left[a_{i+1} ; a_{i+2}, \ldots\right], \quad M_{i}=\lambda_{i}+\Lambda_{i} .
$$

We define

$$
\begin{gathered}
\psi_{i}(x)=M_{i}^{-1}\left[-x^{2}+\left(\Lambda_{i}-\lambda_{i}-1\right) x+\Lambda_{i}\left(1+\lambda_{i}\right)\right], \\
x_{i}=\left(\Lambda_{i}-\lambda_{i}-1\right) / 2, \quad n_{i} \text { is the integer closest to } x_{i} .
\end{gathered}
$$

Then Drobot [1] has shown that $D(\alpha)=\lim \sup _{i \rightarrow \infty} \psi_{i}\left(n_{i}\right)$. In particular, if $\alpha$ has a periodic continued fraction expansion, there are only finitely many choices for $i$ and taking the lim sup reduces to taking the maximum 
of the values $\psi_{i}\left(n_{i}\right)=D_{i}$ of the quadratic polynomial $\psi_{i}(x)$. In view of [3], Theorem 6, p. 1197, it is no restriction to suppose that this expansion is purely periodic and that $a_{0} \geq 1$ :

$$
\alpha=\left[\overline{a_{0} ; a_{1}, \ldots, a_{k-1}}\right], \quad a_{0} \geq 1 .
$$

We extend the periodic sequence

$$
c_{0} ; c_{1}, c_{2}, \ldots=a_{0} ; a_{1}, a_{2}, \ldots, a_{k-1}, a_{0}, a_{1}, \ldots
$$

periodically in the other direction as well, that is,

$$
\ldots c_{-2}, c_{-1}, c_{0} ; c_{1}, c_{2}, \ldots=\ldots a_{0}, a_{1}, \ldots, a_{k-1}, a_{0} ; a_{1}, \ldots, a_{k-1}, a_{0}, a_{1}, \ldots
$$

Then, with $\bar{\lambda}_{i}=\left[0 ; c_{i}, c_{i-1}, \ldots\right], \bar{\Lambda}_{i}=\left[c_{i+1} ; c_{i+2}, \ldots\right]$,

$$
\bar{M}_{i}=\bar{\lambda}_{i}+\bar{\Lambda}_{i}
$$

and similar definitions for $\bar{\psi}_{i}(x), \bar{x}_{i}$ and $\bar{n}_{i}$, one has (see [1], p. 93) $D(\alpha)=$ $\max _{0 \leq i \leq k-1} \bar{\psi}_{i}\left(n_{i}\right)$. In this paper, we only deal with the quadratic irrational case, therefore we may, and do, omit the bar in the above notation without causing any confusion.

We use this method to determine $D(\alpha)$ for $\alpha$ that has a purely periodic continued fraction expansion. Let

$$
\alpha=(A+\sqrt{D}) / B
$$

where

$$
\begin{gathered}
A=p_{k-1}(\alpha)-q_{k-2}(\alpha), \quad B=2 q_{k-1}(\alpha), \\
D=\left(p_{k-1}(\alpha)+q_{k-2}(\alpha)\right)^{2}-4(-1)^{k} ;
\end{gathered}
$$

here $p_{n}(\alpha) / q_{n}(\alpha)$ is the $n$th convergent to $\left[a_{0} ; a_{1}, \ldots, a_{k-1}\right]$. If we set $\Lambda_{i}=$ $(a+\sqrt{D}) / b$, then $\lambda_{i}=(-a+\sqrt{D}) / b$, so that $M_{i}=2 \sqrt{D} / b$ and $2 x_{i}=$ $(2 a-b) / b$.

In particular, if $n_{i}=0$, then

$$
M_{i} D_{i}=\Lambda_{i}+\Lambda_{i} \lambda_{i}=(a+\sqrt{D}) / b+\left(D-a^{2}\right) / b^{2},
$$

so that

$$
\begin{aligned}
D_{i}= & M_{i}^{-1}\left[a / b+\left(D-a^{2}\right) / b^{2}\right]+1 / 2 \\
= & q_{k-1}\left(\Lambda_{i}\right)\left[\left(p_{k-1}\left(\Lambda_{i}\right)+q_{k-2}\left(\Lambda_{i}\right)\right)^{2}-4(-1)^{k}\right]^{-1 / 2} \\
& \times\left[\left(p_{k-1}\left(\Lambda_{i}\right)-q_{k-2}\left(\Lambda_{i}\right)\right) /\left(2 q_{k-1}\left(\Lambda_{i}\right)\right)\right. \\
& \left.+\left(p_{k-1}\left(\Lambda_{i}\right) q_{k-2}\left(\Lambda_{i}\right)-(-1)^{k}\right) / q_{k-1}\left(\Lambda_{i}\right)^{2}\right]+1 / 2 \\
= & {\left[\left(p_{k-1}\left(\Lambda_{i}\right)+q_{k-2}\left(\Lambda_{i}\right)\right)^{2}-4(-1)^{k}\right]^{-1 / 2} } \\
& \times\left[\left(p_{k-1}\left(\Lambda_{i}\right)-q_{k-2}\left(\Lambda_{i}\right)\right) / 2+p_{k-2}\left(\Lambda_{i}\right)\right]+1 / 2 .
\end{aligned}
$$

If $n_{i}=1$, then

$$
M_{i} D_{i}=2 x_{i}-1+\Lambda_{i}+\Lambda_{i} \lambda_{i}=2(a-b) / b+(a+\sqrt{D}) / b+\left(D-a^{2}\right) / b^{2},
$$


so that

$$
\begin{aligned}
D_{i}= & {\left[\left(p_{k-1}\left(\Lambda_{i}\right)+q_{k-2}\left(\Lambda_{i}\right)\right)^{2}-4(-1)^{k}\right]^{-1 / 2} } \\
& \times\left[\left(p_{k-1}\left(\Lambda_{i}\right)-q_{k-2}\left(\Lambda_{i}\right)\right) / 2+p_{k-2}\left(\Lambda_{i}\right)\right. \\
& \left.+\left(p_{k-1}\left(\Lambda_{i}\right)-q_{k-2}\left(\Lambda_{i}\right)-2 q_{k-1}\left(\Lambda_{i}\right)\right)\right]+1 / 2 \\
= & {\left[\left(p_{k-1}\left(\Lambda_{i}\right)+q_{k-2}\left(\Lambda_{i}\right)\right)^{2}-4(-1)^{k}\right]^{-1 / 2} } \\
& \times\left[3\left(p_{k-1}\left(\Lambda_{i}\right)-q_{k-2}\left(\Lambda_{i}\right)\right) / 2\right. \\
& \left.+p_{k-2}\left(\Lambda_{i}\right)-2 q_{k-1}\left(\Lambda_{i}\right)\right]+1 / 2 .
\end{aligned}
$$

Thus, if $n_{i}$ only takes the values 0 and 1 for $i=0, \ldots, k-1$, then $D(\alpha)$ is computed by taking the maximum of the relevant expressions given by equations (A), (B).

For each $k \geq 1$, let $\alpha^{(k)}=\left[\overline{c_{0} ; c_{1}, c_{1}, \ldots, c_{1}}\right]$, with $k$ occurrences of $c_{1}$ and where $c_{0}>c_{1}$. For each $k \geq 1$, we set

$$
\alpha^{(k)}=\Lambda^{(k)}=\left[\overline{c_{0} ; c_{1}, c_{1}, \ldots, c_{1}}\right], \quad \lambda^{(k)}=\left[0 ; \overline{c_{1}, c_{1}, \ldots, c_{1}, c_{0}}\right]
$$

with $k$ occurrences of $c_{1}$.

If $\Lambda^{(k)}=\left(a_{k}+\sqrt{d_{k}}\right) / b_{k}$, then $\lambda^{(k)}=\left(-a_{k}+\sqrt{d_{k}}\right) / b_{k}$, so that

$$
M^{(k)}=\Lambda^{(k)}+\lambda^{(k)}=2 \sqrt{d_{k}} / b_{k} .
$$

In particular, for a fixed $k$, the numerators and the denominators $p_{l}^{(k)}$ and $q_{l}^{(k)}$ of $\alpha^{(k)}$ satisfy

$$
\begin{gathered}
p_{-1}^{(k)}=1, \quad q_{-1}^{(k)}=0, \quad p_{0}^{(k)}=c_{0}, \quad q_{0}^{(k)}=1, \\
p_{l}^{(k)}=c_{1} p_{l-1}^{(k)}+p_{l-2}^{(k)}, \quad q_{l}^{(k)}=c_{1} q_{l-1}^{(k)}+q_{l-2}^{(k)}, \quad 1 \leq l \leq k .
\end{gathered}
$$

Since these are second order linear recurrence equations with constant coefficients, it follows from well known facts (see [4], pp. 121-122, for instance) that

$$
\begin{aligned}
& \left(r_{2}-r_{1}\right) p_{l}^{(k)}=c_{0}\left(r_{2}^{l+1}-r_{1}^{l+1}\right)+r_{2}^{l}-r_{1}^{l}, \\
& \left(r_{2}-r_{1}\right) q_{l}^{(k)}=r_{2}^{l+1}-r_{1}^{l+1}, \quad l=0,1, \ldots, k,
\end{aligned}
$$

where $r_{1}$ and $r_{2}$ satisfy the equation $r^{2}-c_{1} r-1=0$.

Since $a_{k}=p_{k}^{(k)}-q_{k-1}^{(k)}, b_{k}=2 q_{k}^{(k)}$, and $d_{k}=\left(p_{k}^{(k)}+q_{k-1}^{(k)}\right)^{2}+4(-1)^{k}$, it follows that

$$
M_{k}\left(\alpha^{(k)}\right)=\left\{\left(p_{k}^{(k)}+q_{k-1}^{(k)}\right)^{2}+4(-1)^{k}\right\}^{1 / 2} / q_{k}^{(k)},
$$

so that

$$
\begin{aligned}
M_{k}\left(\alpha^{(k)}\right)^{2}= & c_{0}^{2}+4\left(r_{2}^{k+1}-r_{1}^{k+1}\right)^{-2}\left\{c_{0}\left(r_{2}^{k}-r_{1}^{k}\right)\left(r_{2}^{k+1}-r_{1}^{k+1}\right)\right. \\
& \left.+\left(r_{2}^{k}-r_{1}^{k}\right)^{2}+(-1)^{k}\left(r_{2}-r_{1}\right)\right\} \\
= & c_{0}^{2}+4\left(r_{2}^{k+1}-r_{1}^{k+1}\right)^{-2}\left\{c_{0}\left(r_{2}^{2 k+1}+r_{1}^{2 k+1}+(-1)^{k} c_{1}\right)\right. \\
& \left.+\left(r_{2}^{k}-r_{1}^{k}\right)^{2}+(-1)^{k}\left(c_{1}^{2}+4\right)\right\}
\end{aligned}
$$


With the previous notation, we may now write $\alpha^{(k)}=\Lambda^{(k)}=\Lambda_{k}\left(\alpha^{(k)}\right)$ and

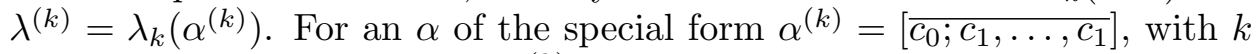
occurrences of $c_{1}$, we have $M\left(\alpha^{(k)}\right)=\max _{0 \leq i \leq k-1} M_{i}$ (see [2], formula (11), p. 29). Since $c_{0}>c_{1}$, we have

$$
\begin{aligned}
M_{k}\left(\alpha^{(k)}\right) & =c_{0}+2\left[0 ; \overline{c_{1}, \ldots, c_{1}, c_{0}}\right] \geq c_{1}+1+2\left[0 ; \overline{c_{1}, \ldots, c_{1}, c_{0}}\right] \\
& >c_{1}+2\left[0 ; \overline{c_{1}, c_{0}, c_{1}, \ldots, c_{1}}\right]=M_{1}\left(\alpha^{(k)}\right) \geq M_{i}\left(\alpha^{(k)}\right) \quad \text { for } i \neq k .
\end{aligned}
$$

Thus,

$$
M\left(\alpha^{(k)}\right)=M_{k}\left(\alpha^{(k)}\right) .
$$

Using (3), it is easy to see that

$$
M\left(\left[\overline{c_{0}}\right]\right)=\left(c_{0}^{2}+4\right)^{1 / 2}, \quad M\left(\left[\overline{c_{0}, c_{1}}\right]\right)=\left(c_{0}^{2}+4 c_{0} / c_{1}\right)^{1 / 2},
$$

and

$$
M\left(\left[\overline{c_{0}, c_{1}, c_{1}}\right]\right)=\left(c_{0}^{2}+4\left(c_{0} c_{1}+1\right) /\left(c_{1}^{2}+1\right)\right)^{1 / 2} .
$$

We observe that for each $k \geq 1, \Lambda^{(k)}-\lambda^{(k)}=c_{0}$, so that $n^{(k)}=\left[c_{0} / 2\right]$, where $n^{(k)}$ is defined to be the integer closest to $\left(\Lambda^{(k)}-\lambda^{(k)}-1\right) / 2$.

Lemma 1. For each $k \geq 1$,

$$
M\left(\alpha^{(k)}\right)\left(4 D\left(\alpha^{(k)}\right)-M\left(\alpha^{(k)}\right)-2\right)= \begin{cases}0 & \text { if } c_{0} \text { is even, } \\ 1 & \text { if } c_{0} \text { is odd. }\end{cases}
$$

Proof. We recall that for $\alpha$ with a periodic continued fraction expansion,

$D(\alpha)=\max _{i} \psi_{i}\left(n_{i}\right)=\max _{i}\left[\left(\lambda_{i}+\Lambda_{i}\right)^{-1}\left\{-n_{i}^{2}+\left(\Lambda_{i}-\lambda_{i}-1\right) n_{i}+\Lambda_{i}\left(1+\lambda_{i}\right)\right\}\right]$.

Since $n_{i}$ is the integer closest to $\left(\Lambda_{i}-\lambda_{i}-1\right) / 2$, the expression for $\psi_{i}\left(n_{i}\right)$ is an increasing function of $\Lambda_{i}$, so that $D(\alpha)=\psi_{k}\left(n_{k}\right)$, where $n_{k}=n^{(k)}$. Also, since $M_{i}=\lambda_{i}+\Lambda_{i}$, we have

$$
\begin{aligned}
M\left(\alpha^{(k)}\right) D\left(\alpha^{(k)}\right) & \\
= & -\left(n^{(k)}\right)^{2}+\left(\Lambda^{(k)}-\lambda^{(k)}-1\right) n^{(k)}+\Lambda^{(k)}\left(\lambda^{(k)}+1\right) \\
= & -\left[c_{0} / 2\right]^{2}+\left(c_{0}-1\right)\left[c_{0} / 2\right]+\left(d_{k}-a_{k}^{2}\right) / b_{k}^{2}+\left(a_{k}+\sqrt{d_{k}}\right) / b_{k} \\
= & -\left[c_{0} / 2\right]^{2}+\left(c_{0}-1\right)\left[c_{0} / 2\right]+a_{k} / b_{k}-a_{k}^{2} / b_{k}^{2}+M\left(\alpha^{(k)}\right) / 2 \\
& +\left(M\left(\alpha^{(k)}\right) / 2\right)^{2},
\end{aligned}
$$

so that

$$
\begin{aligned}
M\left(\alpha^{(k)}\right)\{4 & \left.\left(\alpha^{(k)}\right)-M\left(\alpha^{(k)}\right)-2\right\} \\
& =4\left\{a_{k} / b_{k}-a_{k}^{2} / b_{k}^{2}+\left(c_{0}-1\right)\left[c_{0} / 2\right]-\left[c_{0} / 2\right]^{2}\right\} \\
& =4\left\{c_{0} / 2-\left(c_{0} / 2\right)^{2}+\left(c_{0}-1\right)\left[c_{0} / 2\right]-\left[c_{0} / 2\right]^{2}\right\} \\
& = \begin{cases}0 & \text { if } c_{0} \text { is even, } \\
1 & \text { if } c_{0} \text { is odd. }\end{cases}
\end{aligned}
$$


1. A condition for $M(\alpha)<M(\beta)$ and $D(\alpha)>D(\beta)$. We recall a result of Drobot [1]: $M(\alpha) \leq 4 D(\alpha)-2 \leq M(\alpha)+M(\alpha)^{-1}$ for any real $\alpha$. Thus, with each real $\alpha$, we may associate a constant $k(\alpha)=k \in[0,1]$ such that $4 D(\alpha)-2=M(\alpha)+k M(\alpha)^{-1}$.

Thus, we may define

$$
k(\alpha)=M(\alpha)(4 D(\alpha)-M(\alpha)-2) .
$$

Suppose that $M(\alpha)<M(\beta)$ but $D(\alpha)>D(\beta)$. Now,

$$
\begin{aligned}
D(\alpha)>D(\beta) & \Leftrightarrow 4 D(\alpha)-2>4 D(\beta)-2 \\
& \Leftrightarrow M(\alpha)+k(\alpha) M(\alpha)^{-1}>M(\beta)+k(\beta) M(\beta)^{-1},
\end{aligned}
$$

for some $k(\alpha), k(\beta) \in[0,1]$. If we can find $\alpha$ such that $k(\alpha)=1$ and $\beta$ such that $k(\beta)=0$, then this reduces to

$$
\begin{aligned}
M(\alpha)<M(\beta)<M(\alpha) & +M(\alpha)^{-1} \\
& \Leftrightarrow M(\alpha)^{2}<M(\beta)^{2}<M(\alpha)^{2}+M(\alpha)^{-2}+2 \\
& \Leftrightarrow 0<M(\beta)^{2}-M(\alpha)^{2}<M(\alpha)^{-2}+2 .
\end{aligned}
$$

The existence of such $\alpha, \beta$ is guaranteed by Lemma 1 , since $k(\alpha)=$ $M(\alpha)(4 D(\alpha)-M(\alpha)-2)$, by (4). We shall use (5), (6) to determine some families of such examples. Henceforth, we let $\alpha, \beta$ be such that $M(\alpha)<M(\beta)$ and $D(\alpha)>D(\beta)$. If $\alpha, \beta$ are each of the form $\alpha^{(k)}$, then each of the constants $k(\alpha), k(\beta)$ is either 0 or 1 .

Since $k(\alpha)=k(\beta)$ implies

$$
\begin{aligned}
4 D(\alpha)-2 & =M(\alpha)+k(\alpha) M(\alpha)^{-1} \\
& =M(\alpha)+k(\beta) M(\alpha)^{-1}<M(\beta)+k(\beta) M(\beta)^{-1}=4 D(\beta)-2,
\end{aligned}
$$

we must choose $k(\alpha)=1, k(\beta)=0$. By Lemma 1 , this is equivalent to choosing the largest partial quotient for $\alpha$ to be odd and the largest partial quotient for $\beta$ to be even.

\section{Main results}

THEOREM 1. If $\alpha, \beta$ are both of the form $\left[\overline{c_{0}, c_{1}}\right]$, with $c_{0} \geq c_{1}$, then $M(\alpha)<M(\beta)$ and $D(\alpha)>D(\beta)$ if and only if $(\alpha, \beta) \in\{([\overline{3,1}],[\overline{4,3}]) ;([\overline{5,3}]$, $[\overline{4,1}]) ;([\overline{5,4}],[\overline{4,1}]) ;([\overline{7,3}],[\overline{6,1}])\}$.

Proof. Let $\alpha=\left[\overline{c_{0}, c_{1}}\right], \beta=\left[\overline{c_{0}^{\prime}, c_{1}^{\prime}}\right]$, where $c_{0} \geq c_{1}, c_{0}^{\prime} \geq c_{1}^{\prime}$; we must choose $c_{0}$ to be odd and $c_{0}^{\prime}$ to be even.

If $\left|c_{0}-c_{0}^{\prime}\right| \neq 1$, then $\left|c_{0}-c_{0}^{\prime}\right| \geq 3$. By the expression for $M\left(\left[\overline{c_{0}, c_{1}}\right]\right)$ given before Lemma 1 , if $c_{0}^{\prime} \leq c_{0}-3$,

$$
M(\beta)-M(\alpha)<\left(c_{0}^{\prime}+2\right)-c_{0} \leq-1,
$$


whereas if $c_{0}^{\prime} \geq c_{0}-3$,

$$
M(\beta)-M(\alpha)>c_{0}^{\prime}-\left(c_{0}+2\right) \geq 1 .
$$

This contradicts (5) since $M(\beta)-M(\alpha)<M(\alpha)^{-1}<1$. Thus, $\left|c_{0}-c_{0}^{\prime}\right|=1$.

Case 1: $c_{0}^{\prime}=c_{0}+1$. In this case, (6) reduces to

$$
0<2 c_{0}+1+4\left(\left(c_{0}+1\right) / c_{1}^{\prime}-c_{0} / c_{1}\right)<2+\left(c_{0}^{2}+4 c_{0} / c_{1}\right)^{-1} .
$$

If $c_{1} \geq 2$, then

$$
\begin{aligned}
2 c_{0}+1+4\left(\left(c_{0}+1\right) / c_{1}^{\prime}-c_{0} / c_{1}\right) & \geq 2 c_{0}+1+4\left(\left(c_{0}+1\right) /\left(c_{0}+1\right)-c_{0} / 2\right) \\
& =5>2+\left(c_{0}^{2}+4 c_{0} / c_{1}\right)^{-1} .
\end{aligned}
$$

Thus, $c_{1}=1$ and (7) reduces to

$$
0<-2 c_{0}+1+4\left(c_{0}+1\right) / c_{1}^{\prime}<2+\left(c_{0}^{2}+4 c_{0}\right)^{-1} .
$$

If $c_{1}^{\prime}=1$ or 2 in (8), there is a contradiction. If $c_{1}^{\prime}=3$, the first inequality in (8) is only satisfied when $1 \leq c_{0} \leq 3$. If $c_{0}=1$, then $c_{0}^{\prime}=2$, in contradiction with $c_{1}^{\prime}=3>2$. Since $c_{0}$ is odd, the only example is obtained with $c_{0}=3$. If $c_{1}^{\prime} \geq 4$, we are in conflict with the first inequality of (8), and this case furnishes no further examples.

Thus, the only example in this case is $(\alpha, \beta)=([\overline{3,1}],[\overline{4,3}])$.

Case 2: $c_{0}^{\prime}=c_{0}-1$. In this case, (6) reduces to

$$
0<-2 c_{0}+1+4\left(\left(c_{0}-1\right) / c_{1}^{\prime}-c_{0} / c_{1}\right)<2+\left(c_{0}^{2}+4 c_{0} / c_{1}\right)^{-1} .
$$

If $c_{1}^{\prime} \geq 2$, then

$$
\begin{aligned}
-2 c_{0}+1+4\left(\left(c_{0}+1\right) / c_{1}^{\prime}-c_{0} / c_{1}\right) & \leq-2 c_{0}+1+4\left(\left(c_{0}-1\right) / 2-c_{0} / c_{1}\right) \\
& =-1-4 c_{0} / c_{1}<0 .
\end{aligned}
$$

Thus, $c_{1}^{\prime}=1$ and (9) reduces to

$$
0<2 c_{0}-3-4 c_{0} / c_{1}<2+\left(c_{0}^{2}+4 c_{0}\right)^{-1} .
$$

If $c_{1} \leq 2$, then $2 c_{0}-3-4 c_{0} / c_{1} \leq-3$. If $c_{1} \geq 5$, then $2 c_{0}-3-4 c_{0} / c_{1} \geq 6 c_{0} / 5-$ $3 \geq 3$, since $c_{0} \geq c_{1} \geq 5$. If $c_{1}=3$, then $0<2 c_{0} / 3-3<2+\left(c_{0}^{2}+4 c_{0}\right)^{-1}$, so that $c_{0}=5$ or 7 . If $c_{1}=4$, then $0<c_{0}-3<2+\left(c_{0}^{2}+4 c_{0}\right)^{-1}$, so that $c_{0}=5$.

Thus, the three examples in this case are $(\alpha, \beta)=([\overline{5,3}],[\overline{4,1}]),([\overline{5,4}]$, $[\overline{4,1}])$ and $([\overline{7,3}],[\overline{6,1}])$. This completes the proof. 
Table 1 (Theorem 1)

\begin{tabular}{|c|c|c|c|}
\hline$\xi$ & $M(\xi)$ & $D(\xi)$ & \\
\hline$\overline{3,1}]=(3+\sqrt{21}) / 2$ & $\approx 4.58258$ & $(5.5 / \sqrt{21})+1 / 2$ & \\
\hline$[\overline{4,3}]=(6+\sqrt{48}) / 3$ & $\sqrt{192} / 3 \approx 4$ & $(16 / \sqrt{192})+1 / 2$ & \\
\hline$[\overline{5,3}]=(15+\sqrt{285}) / 6$ & $\sqrt{285} / 3 \approx 5$ & $5 / \sqrt{285})+1 / 2$ & \\
\hline$[\overline{4,1}]=2+\sqrt{8}$ & $\sqrt{32}$ & $(8 / \sqrt{32})+1 / 2$ & $\approx 1.91421$ \\
\hline$[\overline{5,4}]=(5+\sqrt{30}) / 2$ & $\sqrt{480} / 4 \approx 5.47723$ & $(31 / \sqrt{480})+1 / 2$ & $\approx 1$ \\
\hline$[\overline{4,1}]=2+\sqrt{8}$ & $\sqrt{32} \approx 5.65685$ & $(8 / \sqrt{32})+1 / 2$ & $\approx 1.91421$ \\
\hline$[\overline{7,3}]=(21+\sqrt{525}) / 6$ & $\sqrt{525} / 3 \approx 7.63763$ & $(44.5 / \sqrt{525})+1 /$ & 4214 \\
\hline$[\overline{6,1}]=3+\sqrt{15}$ & $\approx 7.74597$ & $(15 / \sqrt{60})+1 / 2$ & $\approx 2.43649$ \\
\hline
\end{tabular}

Theorem 2. If each of $\alpha, \beta$ is of the form $\left[\overline{c_{0}, c_{1}}\right]$ or $\left[\overline{c_{0}, c_{1}, c_{1}}\right], c_{0} \geq$ $c_{1}$, with $\alpha, \beta$ of different forms, then $M(\alpha)<M(\beta)$ and $D(\alpha)>D(\beta)$ if and only if $(\alpha, \beta) \in\{([\overline{3,1}],[\overline{4,3,3}]) ;([\overline{5,1}],[\overline{6,2,2}]) ;([\overline{7,1}],[\overline{8,2,2}])$; $([\overline{5,3,3}],[\overline{4,1}]) ;([\overline{11,2,2}],[\overline{10,1}]) ;([\overline{13,2,2}],[\overline{12,1}])\}$.

The proof of Theorem 2 is similar to that of Theorem 1, and may be found in [5]. Tables similar to Table 1 that verify the results of the various theorems have been omitted.

We observe that $M\left(\alpha^{(k)}\right)=c_{0}+2\left[0, \overline{c_{1}^{(k)}, c_{0}}\right]$ if $c_{0}>c_{1}$. It follows that $\left\{M\left(\alpha^{(2 k)}\right)\right\}_{k \geq 0}$ is an increasing sequence, that $\left\{M\left(\alpha^{(2 k+1)}\right)\right\}_{k \geq 0}$ is a decreasing sequence, and that $M\left(\alpha^{(2 m+1)}\right)>M\left(\alpha^{(2 n)}\right)$ for any choice of $m, n \geq 0$. Furthermore,

$$
\lim _{n \rightarrow \infty} M\left(\alpha^{(n)}\right)=c_{0}+2\left[0, \overline{c_{1}}\right]=\left(c_{0}-c_{1}\right)+\sqrt{c_{1}^{2}+4} .
$$

Theorem 3. For any $n \geq 2,(\alpha, \beta) \in\left\{\left([\overline{3,1}],\left[\overline{4,3^{(n)}}\right]\right) ;\left([\overline{5,1}],\left[\overline{6,2^{(n)}}\right]\right)\right.$; $\left.\left([\overline{7,1}],\left[\overline{8,2^{(n)}}\right]\right) ;\left(\left[\overline{5,3^{(n)}}\right],[\overline{4,1}]\right) ; \quad\left(\left[\overline{11,2^{(n)}}\right],[\overline{10,1}]\right) ; \quad\left(\left[\overline{13,2^{(n)}}\right],[\overline{12,1}]\right)\right\}$ satisfy the conditions $M(\alpha)<M(\beta), D(\alpha)>D(\beta)$.

Proof. In view of the observations made above, we need to determine only $M\left(\overline{\left[c_{0}, c_{1}^{(3)}\right]}\right)$. The result then follows from the inequalities $\left.M\left(\overline{\left[c_{0}, c_{1}^{(2)}\right.}\right]\right)$ $\left.\left.<M\left(\overline{\left[c_{0}, c_{1}^{(n)}\right]}\right]\right)<M\left(\overline{\left[c_{0}, c_{1}^{(3)}\right.}\right]\right)$ for every $n \geq 4$, and from Theorem 2, Lemma 1 and (6). This completes the proof.

\begin{tabular}{cccccc}
\hline$\xi_{1}$ & $\xi_{2}^{(n)}$ & $\left\{M\left(\xi_{1}\right)\right\}^{2}$ & $\left\{M\left(\xi_{2}^{(2)}\right)\right\}^{2}$ & $\left\{M\left(\xi_{2}^{(3)}\right)\right\}^{2}$ & $\left\{\lim _{n \rightarrow \infty} M\left(\xi_{2}^{(n)}\right)\right\}^{2}$ \\
\hline$[\overline{3,1}]$ & {$\left[\overline{4,3^{(n)}}\right]$} & 21.0 & 21.2 & $21 . \overline{21}$ & $21.21110 \ldots$ \\
{$[\overline{5,1}]$} & {$\left[\overline{6,2^{(n)}}\right]$} & 45.0 & 46.4 & $46 . \overline{6}$ & $46.62741 \ldots$ \\
{$[\overline{7,1}]$} & {$\left[\overline{\left[8,2^{(n)}\right.}\right]$} & 77.0 & 77.6 & 78.0 & $77.94112 \ldots$ \\
{$[\overline{4,1}]$} & {$\left[\overline{\left[, 3^{(n)}\right.}\right]$} & 32.0 & 31.4 & $31 . \overline{42}$ & $31.42220 \ldots$ \\
{$[\overline{10,1}]$} & {$\left[\overline{11,2^{(n)}}\right]$} & 140.0 & 139.4 & 140.0 & $139.91168 \ldots$ \\
{$[\overline{12,1}]$} & {$\left[\overline{13,2^{(n)}}\right]$} & 192.0 & 190.6 & $191 . \overline{3}$ & $191.22539 \ldots$ \\
\hline
\end{tabular}


THEOREM 4. If each of $\alpha, \beta$ is of the form $\left[\overline{c_{0}, c_{1}, c_{1}}\right], c_{0} \geq c_{1}$, then

$$
M(\alpha)<M(\beta) \Rightarrow D(\alpha)<D(\beta) .
$$

Proof. If $\alpha=\left[\overline{c_{0}, c_{1}, c_{1}}\right], c_{0} \geq c_{1}$ and $\beta=\left[\overline{c_{0}^{\prime}, c_{1}^{\prime}, c_{1}^{\prime}}\right], c_{0}^{\prime} \geq c_{1}^{\prime}$, then $c_{0}$ is odd and $c_{0}^{\prime}$ is even. If $c_{0}^{\prime} \geq c_{0}+1$, then

$$
\begin{aligned}
M(\beta)^{2}-M(\alpha)^{2} & =c_{0}^{\prime 2}-c_{0}^{2}+4\left\{\left(c_{0}^{\prime} c_{1}^{\prime}+1\right) /\left(c_{1}^{\prime 2}+1\right)-\left(c_{0} c_{1}+1\right) /\left(c_{1}^{2}+1\right)\right\} \\
& \geq 2 c_{0}+1+4\left\{1-\left(c_{0}+1\right) / 2\right\}=3,
\end{aligned}
$$

in contradiction to $(6)$.

$$
\begin{aligned}
& \text { If } c_{0}^{\prime} \leq c_{0}-1 \text {, then } \\
& \begin{aligned}
M(\beta)^{2}-M(\alpha)^{2} & =c_{0}^{\prime 2}-c_{0}^{2}+4\left\{\left(c_{0}^{\prime} c_{1}^{\prime}+1\right) /\left(c_{1}^{2}+1\right)-\left(c_{0} c_{1}+1\right) /\left(c_{1}^{2}+1\right)\right\} \\
& \leq-2 c_{0}+1+4\left\{\left(c_{0}^{\prime}+1\right) / 2-1\right\} \\
& \leq-2 c_{0}+1+4\left(c_{0} / 2-1\right)=-3,
\end{aligned}
\end{aligned}
$$

so that $M(\beta)<M(\alpha)$. This completes the proof.

V. Drobot [1] observed that $k(\xi)=M(\xi)(4 D(\xi)-M(\xi)-2)=0$ or 1 depending on whether $A$ is even or odd for $\xi=\left[1_{m(1)}, A, 1_{m(2)}, A, 1_{m(3)}, A, \ldots\right]$, where $A>3$ and $\left\{m_{j}\right\}_{j \geq 1}$ is a non-decreasing sequence of integers tending to infinity. Thus, by the results of Section 1, in order to obtain a counterexample, $A$ must be chosen to be odd if $\alpha=\xi$ and even if $\beta=\xi$.

Since $\left\{m_{j}\right\}_{j \geq 1}$ is a non-decreasing sequence of integers tending to infinity, $M(\xi)$ is computed by taking the limit superior of a sequence with leading partial quotient $A$. In fact,

(10) $\quad M(\xi)$

$$
\begin{aligned}
& =\limsup _{i \rightarrow \infty}\left(\left[A, 1_{m(i)}, A, 1_{m(i+1)}, \ldots\right]+\left[0,1_{m(i-1)}, A, 1_{m(i-2)}, \ldots, A, 1_{m(1)}\right]\right) \\
& =A+2[0, \overline{1}] \\
& =A+(\sqrt{5}-1), \quad \text { for any } A \geq 1 .
\end{aligned}
$$

Theorem 5. If each of $\alpha, \beta$ is of the form $\left[1_{m(1)}, A, 1_{m(2)}, A, 1_{m(3)}\right.$, $A, \ldots]$, where $A>3$ and $\left\{m_{j}\right\}_{j \geq 1}$ is a non-decreasing sequence of integers tending to infinity, then

$$
M(\alpha)<M(\beta) \Rightarrow D(\alpha)<D(\beta) .
$$

Proof. By $(10), M(\alpha) \neq M(\beta) \Rightarrow M(\beta)-M(\alpha) \geq 1>M(\alpha)^{-1}$, which contradicts (5). This completes the proof.

Theorem 6. If $v=\left[\overline{c_{0}, c_{1}}\right]$, where $c_{0} \geq c_{1}, \xi=\left[1_{m(1)}, A, 1_{m(2)}, A, 1_{m(3)}\right.$, $A, \ldots], A>3$ and $\left\{m_{j}\right\}_{j \geq 1}$ is a non-decreasing sequence of integers tending to infinity, and $(v, \xi)$ satisfies the inequality $(M(v)-M(\xi))(D(v)-D(\xi))<$ 0 , then $\left(c_{0}, c_{1}\right) \in\left\{\left(c_{0}, 9\right), 9 \leq c_{0} \leq 69, c_{0}\right.$ odd $;\left(c_{0}, 10\right), 11 \leq c_{0} \leq 25, c_{0}$ 
odd; $\left(c_{0}, 11\right), 11 \leq c_{0} \leq 17, c_{0}$ odd $;\left(c_{0}, 8\right), 8 \leq c_{0} \leq 72, c_{0}$ even; $\left(c_{0}, 7\right)$, $8 \leq c_{0} \leq 20, c_{0}$ even; $\left(c_{0}, 6\right), 6 \leq c_{0} \leq 10, c_{0}$ even; $\left.(13,12) ;(6,5)\right\}$, and $A=c_{0}-1$.

The proof of Theorem 6 is similar to that of Theorem 1, and may be found in [5].

Theorem 7. If $v=\left[\overline{c_{0}, c_{1}, c_{1}}\right]$, where $c_{0} \geq c_{1}, \xi=\left[1_{m(1)}, A, 1_{m(2)}, A, \ldots\right]$, $A>3,\left\{m_{j}\right\}_{j \geq 1}$ is a non-decreasing sequence of integers tending to infinity, and $(v, \xi)$ satisfies the inequality $(M(v)-M(\xi))(D(v)-D(\xi))<0$, then $\left(c_{0}, c_{1}\right) \in\left\{\left(c_{0}, 9\right), 9 \leq c_{0} \leq 59, c_{0}\right.$ odd $;\left(c_{0}, 10\right), 11 \leq c_{0} \leq 25, c_{0}$ odd; $\left(c_{0}, 11\right), 11 \leq c_{0} \leq 17, c_{0}$ odd; $\left(c_{0}, 8\right), 8 \leq c_{0} \leq 98, c_{0}$ even; $\left(c_{0}, 7\right)$, $8 \leq c_{0} \leq 22, c_{0}$ even; $\left(c_{0}, 6\right), 6 \leq c_{0} \leq 10, c_{0}$ even; $\left.(13,12) ;(6,5)\right\}$, and $A=c_{0}-1$.

The proof of Theorem 7 is similar to that of Theorem 1, and is omitted.

We recall that $M\left(\alpha^{(k)}\right)=c_{0}+2\left[0, \overline{c_{1}^{(k)}, c_{0}}\right]$ if $c_{0}>c_{1}$, that $\left\{M\left(\alpha^{(2 k)}\right)\right\}_{k \geq 0}$ is an increasing sequence, that $\left\{M\left(\alpha^{(2 k+1)}\right)\right\}_{k \geq 0}$ is a decreasing sequence, and that $M\left(\alpha^{(2 m+1)}\right)>M\left(\alpha^{(2 n)}\right)$ for any choice of $m, n \geq 0$. Furthermore,

$$
\lim _{n \rightarrow \infty} M\left(\alpha^{(n)}\right)=c_{0}+2\left[0, \overline{c_{1}}\right]=c_{0}-c_{1}+\sqrt{c_{1}^{2}+4} .
$$

Theorem 8. If $v=\left[\overline{c_{0}, c_{1}^{(n)}}\right]$, where $c_{0} \geq c_{1}, n \geq 1$, and $\xi=\left[1_{m(1)}, A\right.$, $\left.1_{m(2)}, A, 1_{m(3)}, A, \ldots\right], A>3$ and $\left\{m_{j}\right\}_{j \geq 1}$ is a non-decreasing sequence of integers tending to infinity, and $(v, \xi)$ satisfies the inequality $(M(v)-$ $M(\xi))(D(v)-D(\xi))<0$, then $\left(c_{0}, c_{1}\right) \in\left\{\left(c_{0}, 9\right), 9 \leq c_{0} \leq 59, c_{0}\right.$ odd; $\left(c_{0}, 10\right), 11 \leq c_{0} \leq 25, c_{0}$ odd; $\left(c_{0}, 11\right), 11 \leq c_{0} \leq 17, c_{0}$ odd; $\left(c_{0}, 8\right)$, $8 \leq c_{0} \leq 98, c_{0}$ even; $\left(c_{0}, 7\right), 8 \leq c_{0} \leq 22, c_{0}$ even $;\left(c_{0}, 6\right), 6 \leq c_{0} \leq 10, c_{0}$ even; $(13,12) ;(6,5)\}$, and $A=c_{0}-1$.

Proof. In view of the observations made above, the theorem follows from the inequalities $M\left(\left[\overline{c_{0}, c_{1}^{(2)}}\right]\right)<M\left(\left[\overline{c_{0}, c_{1}^{(n)}}\right]\right)<M\left(\left[\overline{c_{0}, c_{1}^{(1)}}\right]\right)$ for every $n \geq 3$, and from Theorems 6 and 7 .

Theorem 9. For each fixed value of $n \geq 3$, there exists $\alpha, \beta$ of the form $\overline{\left[c_{0}, c_{1}^{(n)}\right]}$, where $c_{0}>c_{1}$, such that $(M(\alpha)-M(\beta))(D(\alpha)-D(\beta))<0$.

The proof of Theorem 9 is similar to that of Theorem 1, but the computation is more intricate and has been omitted here. It may be noted that we must choose $\alpha, \beta$ of the form $\left.\left[\overline{c_{0}+1, c_{1}^{(n)}}\right], \overline{c_{0}, 1^{(n)}}\right]$, where $c_{0} \geq c_{1}$ and the largest partial quotient for $\alpha$ is odd.

We observe that if $\alpha=\alpha^{(n)}, \beta=\beta^{(n)}$ are chosen as in Theorem 9 and if $(M(\alpha)-M(\beta))(D(\alpha)-D(\beta))<0$ for all $n \geq N$, then this inequality must also hold for $\alpha=\lim _{n \rightarrow \infty} \alpha^{(n)}, \beta=\lim _{n \rightarrow \infty} \bar{\beta}^{(n)}$. This reduces to the choice $\alpha=\lim _{n \rightarrow \infty}\left[\overline{c_{0}, 1^{(n)}}\right], \beta=\lim _{n \rightarrow \infty}\left[\overline{c_{0}+1, c_{1}^{(n)}}\right]$, where $c_{0} \geq c_{1}, c_{0}$ odd. 
Thus, $M(\alpha)=c_{0}-1+\sqrt{5}$ and $M(\beta)=c_{0}-c_{1}+1+\sqrt{c_{1}^{2}+4}$, so that $c_{0} \geq 5$ since $c_{0}=3$ gives $M(\beta)^{2}-M(\alpha)^{2} \geq(2+\sqrt{8})^{2}-(2+\sqrt{5})^{2}>2+M(\alpha)^{-2}$. A simple computation yields $5 \leq c_{1} \leq 8$, and for each such $c_{1}$, the upper limits for $c_{0}$ are $5,9,21$, and 97 , respectively.

If, on the other hand, we choose $\alpha=\lim _{n \rightarrow \infty}\left[\overline{c_{0}+1, c_{1}^{(n)}}\right], \quad \beta=$ $\lim _{n \rightarrow \infty}\left[\overline{c_{0}, 1^{(n)}}\right], c_{1} \leq c_{0}, c_{0}$ even, a simple computation yields $9 \leq c_{1} \leq 12$, and for each such $c_{1}$, the upper limits for $c_{0}$ are 58,24, 16, and 12, respectively.

In particular, since $M\left(\left[\overline{7,1^{(4)}}\right]\right)^{2}=67.4 \leq M\left(\left[\overline{7,1^{(n)}}\right]\right)^{2} \leq 69=$ $M\left(\left[\overline{7,1^{(3)}}\right]\right)^{2}$ for each $n \geq 3$, and $M\left(\left[\overline{8,6^{(4)}}\right]\right)^{2}<69.298220641 \leq M\left(\left[\overline{8,6^{(n)}}\right]\right)^{2}$ $\leq 69.298245614<M\left(\left[\overline{8,6^{(3)}}\right]\right)^{2}$ for each $n \geq 3$, there exists $\alpha^{(n)}, \beta^{(n)}$ for each $n \geq 3$ such that $\left(M\left(\alpha^{(n)}\right)-M\left(\beta^{(n)}\right)\right)\left(D\left(\alpha^{(n)}\right)-D\left(\beta^{(n)}\right)\right)<0$.

We end this paper with a table of values of Markov and dispersion constants of the first sixteen numbers in the Markov spectrum. This list, provided by the referee, contains the first sixteen Markov numbers $u_{n}$, the corresponding real irrational number $\alpha_{n}, \Delta_{n}:=9 u_{n}^{2}-4, M\left(\alpha_{n}\right)$, and $D\left(\alpha_{n}\right)$, and yields another twenty seven counterexamples and suggests the abundance of such.

Table 2. Smallest Markov constants with corresponding dispersion constants

\begin{tabular}{llllcc}
\hline$n$ & $u_{n}$ & \multicolumn{1}{c}{$\Delta_{n}$} & \multicolumn{1}{c}{$\alpha_{n}$} & \multicolumn{1}{c}{$M\left(\alpha_{n}\right)$} & $D\left(\alpha_{n}\right)$ \\
\hline 1 & 1 & 5 & {$[0, \overline{1}]$} & $2.2360679 \ldots$ & $1.1708203 \ldots$ \\
2 & 2 & 32 & {$[0, \overline{2}]$} & $2.8284271 \ldots$ & $1.2071067 \ldots$ \\
3 & 5 & 221 & {$\left[0, \overline{2_{2}, 1_{2}}\right]$} & $2.9732137 \ldots$ & $1.2735737 \ldots$ \\
4 & 13 & 1517 & {$\left[0, \overline{2_{2}, 1_{4}}\right]$} & $2.9960526 \ldots$ & $1.2830816 \ldots$ \\
5 & 29 & 7565 & {$\left[0, \overline{2_{4}, 1_{2}}\right]$} & $2.9992071 \ldots$ & $1.2760671 \ldots$ \\
6 & 34 & 10400 & {$\left[0, \overline{2_{2}, 1_{6}}\right]$} & $2.9994232 \ldots$ & $1.2844645 \ldots$ \\
7 & 89 & 71285 & {$\left[0, \overline{2_{2}, 1_{8}}\right]$} & $2.9999158 \ldots$ & $1.2846662 \ldots$ \\
8 & 169 & 257045 & {$\left[0, \overline{2_{6}, 1_{2}}\right]$} & $2.9999766 \ldots$ & $1.2761401 \ldots$ \\
9 & 194 & 338720 & {$\left[0, \overline{2_{2}, 1_{2}, 2_{2}, 1_{4}}\right]$} & $2.9999822 \ldots$ & $1.2835097 \ldots$ \\
10 & 233 & 488597 & {$\left[0, \overline{2_{2}, 1_{10}}\right]$} & $2.9999877 \ldots$ & $1.2846956 \ldots$ \\
11 & 433 & 1687397 & {$\left[0, \overline{2_{2}, 1_{2}, 2_{4}, 1_{2}}\right]$} & $2.9999964 \ldots$ & $1.2763673 \ldots$ \\
12 & 610 & 3348896 & {$\left[0, \overline{2_{2}, 1_{12}}\right]$} & $2.9999982 \ldots$ & $1.2846999 \ldots$ \\
13 & 985 & 8732021 & {$\left[0, \overline{2_{8}, 1_{2}}\right]$} & $2.9999993 \ldots$ & $1.2761423 \ldots$ \\
14 & 1325 & 15800621 & {$\left[0, \overline{2_{2}, 1_{4}, 2_{2}, 1_{6}}\right]$} & $2.9999996 \ldots$ & $1.2845284 \ldots$ \\
15 & 1597 & 22953677 & {$\left[0, \overline{2_{2}, 1_{14}}\right]$} & $2.9999997 \ldots$ & $1.2847005 \ldots$ \\
16 & 2897 & 75533477 & {$\left[0, \overline{2_{2}, 1_{2}, 2_{2}, 1_{2}, 2_{2}, 1_{4}}\right]$} & $2.9999999 \ldots$ & $1.2835116 \ldots$ \\
\hline
\end{tabular}

Acknowledgement. The basic results of this work form a part of the author's thesis done while at SUNY, Buffalo. I wish to thank Professor Thomas W. Cusick for his patient and invaluable guidance in this work, and for the references [1] and [3], without which this would not have been. 
I am also thankful to the referee for the innumerable suggestions that have made this work a little more readable and also for the list of counterexamples that appear at the end.

\section{References}

[1] V. Drobot, On dispersion and Markov constants, Acta Math. Hungar. 47 (1986), 89-93.

[2] J. F. Koksma, Diophantische Approximationen, Springer, Berlin 1936.

[3] H. Niederreiter, On a measure of denseness for sequences, in: Topics in Classical Number Theory, Vol. 2, Colloq. Math. Soc. János Bolyai 34, North-Holland, Amsterdam 1984, 1163-1208.

[4] I. Niven and H. S. Zuckerman, An Introduction to the Theory of Numbers, 4th ed., Wiley, 1980.

[5] A. Tripathi, Topics in number theory, Thesis, State University of New York at Buffalo, Department of Mathematics, 1989.

Current address:

DEPARTMENT OF MATHEMATICS

FAIRMONT STATE COLLEGE

FAIRMONT, WEST VIRGINIA 26554

U.S.A.

\author{
DEPARTMENT OF MATHEMATICS \\ INDIAN INSTITUTE OF TECHNOLOGY \\ HAUZ KHAS, DELHI 110016 \\ INDIA
}

